\title{
A Rare Case of Synchronous Bilateral Pleomorphic Adenoma of the Parotid Gland
}

\author{
Ricciardiello Filippo, Papa Angelo, Oliva Flavia, Pianese Annalisa, Abate Teresa, \\ Mesolella Massimo \\ Department of Otolaryngology-Head and Neck Surgery, University of Naples "Federico II", Naples, Italy \\ Email: filipporicciardiello@virgilio.it
}

Received 18 May 2014; revised 17 June 2014; accepted 13 July 2014

Copyright (C 2014 by authors and Scientific Research Publishing Inc. This work is licensed under the Creative Commons Attribution International License (CC BY). http://creativecommons.org/licenses/by/4.0/ cc) (7) Open Access

\begin{abstract}
Multiple parotid gland tumors are unusual. Warthin's tumor is the most represented neoplasm in this condition. We present a case of bilateral synchronous pleomorphic adenoma affecting the parotid glands in a 28-year-old woman. Clinically, the lesions were similar to solitary pleomorphic adenoma. Both lesions were diagnosed by fine-needle aspiration cytology. A total parotidectomy for left tumor and surgical enucleation for the right tumor, respectively, were performed with no facial nerves injury. A review of the pertinent literature is included.
\end{abstract}

\section{Keywords}

Multiple Parotid Gland Tumors, Pleomorphic Adenoma, Synchronous, Total Parotidectomy

\section{Introduction}

Salivary gland tumors represent approximately 3\% of all head and neck tumors. About 70\% - 80\% of these neoplasms occur in parotid gland [1]; clinical presentation usually includes an unilateral, growing nodule associated with different symptoms depending on the tumor type. Multiple parotid gland tumors (MPGT) are unusual; they amount approximately to 3.4\% of all parotid gland tumors [2]. In 1996, Seifert and Donath reviewed the nomenclature of the multiple salivary gland tumors, differentiating three groups: the bilateral tumors, the unilateral multifocal ones and the combination of bilateral tumors with unilateral multifocal tumors [3]. Another parameter to consider is the chronology of diagnosis; they may occur synchronously or metachronously, excluding postoperative recurrent neoplasms and metastatic lesions from other primary malignant tumors. MPGT occur in life later than solitary tumors (average 61 - 68 years) [4], and are more common in men than in women (5.9:1) [2]. Turnbull and Frazell indicated that MPGT can arise after the radiotherapy of benign lesions. They also reported that $79.2 \%$ of bilateral parotid tumors (BPT) are represented by Warthin's tumors whereas pleomorphic adeno-

How to cite this paper: Filippo, R., Angelo, P., Flavia, O., Annalisa, P., Teresa, A. and Massimo, M. (2014) A Rare Case of Synchronous Bilateral Pleomorphic Adenoma of the Parotid Gland. Case Reports in Clinical Medicine, 3, 387-391. 
ma (PA) is the second most common BPT [5]. We report a case of a young woman with synchronous bilateral pleomorphic adenoma of the parotid gland.

\section{Case Report}

In March 2011, a 28-year-old caucasian woman was admitted to our ENT department, at the University Federico II of Naples (Italy), with one-year history of a painless nodules in both pre-auricular areas, that had slowly enlarged. Physical examination revealed a solid mass at the left parotid gland larger than the right parotid one, both well defined, hard-elastic, with no fixation to the adjacent anatomic structures. There weren't facial nerves palsy or cervical lymphadenopathies.

The patient underwent to fine-needle aspiration cytology (FNAC) of the parotid glands lesions, that showed round and oval cells with regular nucleus and no malignant elements, suggestive for pleomorphic adenoma. A magnetic resonance scan showed a nodule, measuring at the largest diameters $1.0 \times 1.0 \times 1.6 \mathrm{~cm}$, arising the deep lobe of the right parotid gland, and a mass measuring $2.3 \times 1.9 \times 2.0 \mathrm{~cm}$ originating from the deep lobe of the left parotid gland. Both lesions appeared iso-hypointense on T1-weighted images (Figure 1A); on T2weighted images the right parotid gland nodule showed marked high signal intensity whereas the left parotid gland mass appeared unevenly hyperintense (Figure 1B; Figure 2). After intravenous gadolinium injection,

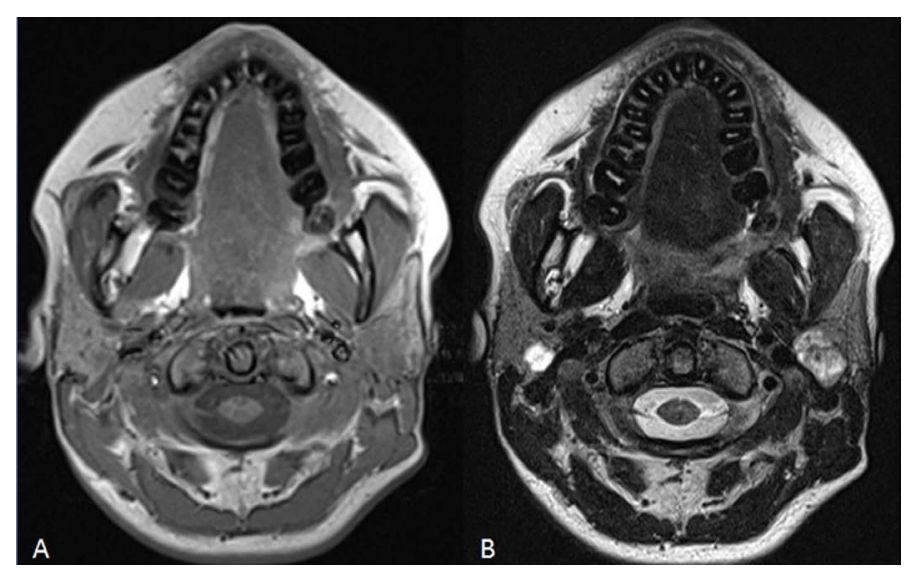

Figure 1. Axial MR images demonstrating the presence of a mass both in the right and in the left parotid gland; both tumors show an iso-hypointense signal on T1-weighted image (A) and a hyperintense signal on T2weighted image (B).



Figure 2. Axial (A) and coronal (B) fat-suppressed T2-weighted MR image demonstrating a marked high signal intensity well-defined margin mass in the superficial lobe of the right parotid gland and an inhomogeneous high signal intensity mass with lobulated margins in the deep lobe of the left parotid gland. 
the right parotid gland nodule showed peripheral enhancement while the left parotid gland mass showed inhomogeneous contrast enhancement (Figure 3); in both cases no evidence of invasion of adjacent soft tissues or local lymph nodes enlargement were observed.

Two-time surgery was indicated to avoid bilateral facial nerve palsy. In May 2011, the left tumor was excised by total parotidectomy with facial nerve preservation under general anesthesia. Six months later no recurrence or facial palsy of the left side were observed. In February 2012, surgical enucleation of the right tumor was performed preserving the fibrous capsule integrity with no facial injury. We chose this approach in relation to the early age of our patient and the small size of the right lesion. Histopathology exam confirmed the diagnosis of pleomorphic adenoma in both specimens (Figure 4). One year after the second surgery, no recurrence was observed in both parotid areas and no neural dysfunction was reported.

\section{Discussion}

According to the literature, MPGT is an unusual condition, usually described in large studies. In 1969, Turnbull and Frazell reported 32 cases on 1837 patients [5] and in 2004 Yu et al. reported 69 cases on 2055 patients in 40 years [2]. In these studies, it is possible to disclose 55 cases of BPT and only 7 are pleomorphic adenoma.

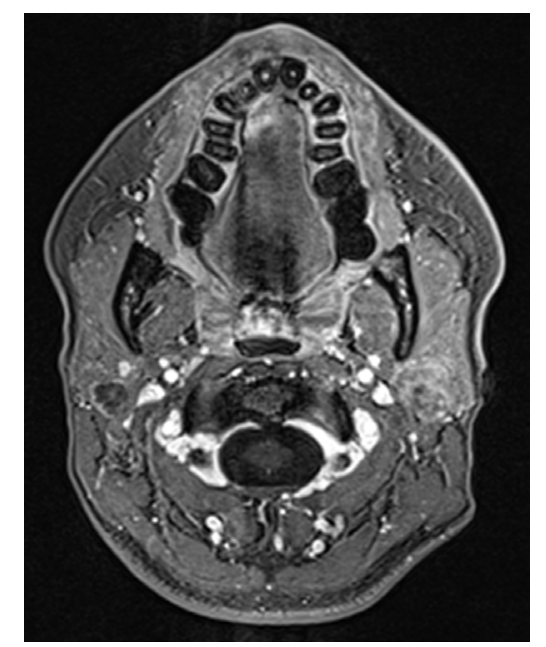

Figure 3. Axial gadolinium-enhanced fat-suppressed T1-weighted image demonstrating peripheral enhancement in the right parotid gland mass and inhomogeneous enhancement in the left parotid gland mass.

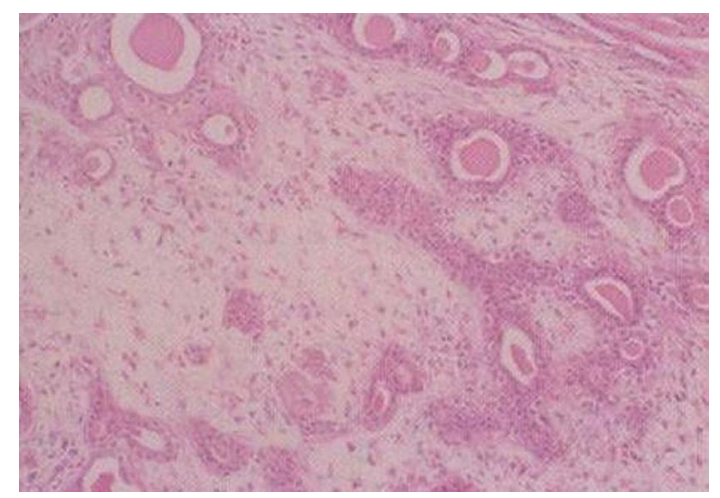

Figure 4. Histopathologic specimen of the left parotid gland mass that shows epithelial cells in a myxoid matrix, confirming the diagnosis of pleomorphic adenoma. 
Classification of MPGT is controversial. In 1996, Seifert and Donath reviewed the nomenclature of these tumors with regard to their localization, classifying them as: bilateral parotid tumours, unilateral multifocal parotid tumours and the combination of bilateral parotid tumours with unilateral multifocal parotid tumours [3]. As regard the chronology, classification is not clear; some authors indicate an interval of 6 months or more from the diagnosis of the first tumor, to define a second tumor as metachronous [6]. However, this parmeter presents some problems related to the diagnosis because the identification of a lesion depends on dimensions and localization of the tumor (superficial or deep lobe of the gland), and on presence or not of symptoms. For example, early tumors, with no symptoms, located in the salivary gland deep lobe can go unnoticed.

Yu et al. [2] reported that synchronous lesions affect men almost 6 times more frequently than women.

Tumors with bilateral involvement present with high frequence an histopathology exam that show a Warthin's tumour (79.2\% to $92.5 \%$ of cases) [5], the second neoplasm more represented is pleomorphic adenoma, representing approximately $6 \%$ of the tumors [1].

Irradiation of head and neck is the most presumable causative factor associated with the development of two pleomorphic adenomas in the parotid gland [7]. In 1983, it was reported an increased incidence of salivary gland tumors after the atomic bomb explosions in Japan and the exposure effects were indicated to be both cumulative and dose dependent [8].

It has been reported that synchronous or metachronous multifocal Warthin's tumors amount $5 \%-20 \%$ of all Warthin's tumors [9]. In these cases, it could be recommended to use ultrasonography as a screening exam to disclose a tumor of the controlateral gland. Yu et al. used this exam as a screening tool for MPGT, to choice surgical approach and during the postoperative follow-up [2]. Ultrasonography can help to perform FNAC of these lesions; this evaluation is essential to exclude a malignancy that could affect the surgical approach to these patients [10]. We did not perform ultrasonography because the lesions were easy to detect and to biopsy. Anyway cross-sectional imaging modalities such as CT (Computed Tomography) and MRI (Magnetic Resonance Imaging) are recommended to detect small lesions and evaluate lymph nodes status, tumor extension and his relationships with neighbouring structures [11]. At CT imaging pleomorphic adenoma can appear as smooth margins tumor, a small spherical tumor or as a lobulated large tumor with inhomogeneous pattern of contrast enhancement in the majority of cases; however the role of CT in assessment of salivary gland tumors is limited except for the evaluation of cortical mandibular involvement and the detection of salivary gland stones. At MR imaging pleomorphic adenoma typically shows an intermediate-low signal on T1-weighted images and a high signal, usually inhomogeneous, on T2-weighted images with inhomogeneous enhancement on contrast-enhanced T1-weighted images; some lesions can also be detected as cystic-appearing nodules with peripheral enhancement. T2 hypointensity of a parotid tumor has been reported as an indicator for malignancy [12]. MR imaging is superior to CT to estimate tumor characteristics and extension, particularly perineural spread [11].

The prevailing treatment for MPGT is the surgical approach similarly to that indicated for solitary tumors; according to the literature we performed a total parotidectomy on the left side and a tumor enucleation on the right side with identification, dissection and preservation of facial nerve branches on both sides. During the surgery care should be taken in order to prevent that tumoral cell islands might be lost on surgical excision margin [13] [14]; for this reason if the tumor is a PA, such as in this case, surgery must preserve the fibrous capsule integrity. Using this surgical approach, no recurrence has been documented in the literature up to now. In case of FNAC or histopathology exam positive for high-grade malignancies, postoperative radiotherapy is usually recommended.

\section{Conclusion}

Bilateral tumors of the parotid gland are not an usual condition, in particular pleomorphic adenoma amounts approximately to $6 \%$ of the BPT. A diagnosis of Warthin's tumor should always be suspected. Our clinical experience shows that a right preoperative assessment and a surgical treatment based on histology are advisable for a proper management. If these conditions are respected, risk of recurrence or facial nerve injury is very low.

\section{References}

[1] Silva, S.J., Costa Jr., G.T., Brant Filho, A.C., et al. (2006) Metachronous Bilateral Pleomorphic Adenoma of the Parotid Gland. Oral Surgery, Oral Medicine, Oral Pathology, Oral Radiology, and Endodontology, 101, 333-338. http://dx.doi.org/10.1016/j.tripleo.2005.07.025 
[2] Yu, G.Y., Ma, D.Q., Zhang, Y., et al. (2004) Multiple Primary Tumours of the Parotid Gland. International Journal of Oral and Maxillofacial Surgery, 33, 531-534. http://dx.doi.org/10.1016/j.ijom.2004.03.010

[3] Seifert, G. and Donath, K. (1996) Multiple Tumours of the Salivary Glands: Terminology and Nomenclature. European Journal of Cancer Part B: Oral Oncology, 32B, 3-7. http://dx.doi.org/10.1016/0964-1955(95)00063-1

[4] Ethunandan, M., Pratt, C.A. and Macpherson, D.W. (2002) Changing Frequency of Parotid Neoplasms-Analysis of 560 Tumours Treated in a District General Hospital. Annals of the Royal College of Surgeons of England, 84, 1-6.

[5] Turnbull, A.D. and Frazell, E.L. (1969) Multiple Tumors of the Major Salivary Glands. The American Journal of Surgery, 118, 787-789. http://dx.doi.org/10.1016/0002-9610(69)90230-X

[6] Ethunandan, M., Pratt, C.A., Morrison, A., et al. (2006) Multiple Synchronous and Metachronous Neoplasms of the Parotid Gland: The Chichester Experience. British Journal of Oral and Maxillofacial Surgery, 44, 397-401. http://dx.doi.org/10.1016/j.bjoms.2005.08.015

[7] Nagler, R.M. and Laufer, D. (1999) Synchronous Pleomorphic Adenomas of the Major Salivary Glands: A Case Report. Oral Surgery, Oral Medicine, Oral Pathology, Oral Radiology, and Endodontology, 87, 735-737. http://dx.doi.org/10.1016/S1079-2104(99)70171-X

[8] Takeichi, N., Hirose, F., Yamamoto, H., et al. (1983) Salivary Gland Tumors in Atomic Bomb Survivors, Hiroshima, Japan. II. Pathologic Study and Supplementary Epidemiologic Observations. Cancer, 52, 377-385. http://dx.doi.org/10.1002/1097-0142(19830715)52:2<377::AID-CNCR2820520232>3.0.CO;2-9

[9] Tanaka, S., Tabuchi, K., Oikawa, K., et al. (2007) Synchronous Unilateral Parotid Gland Neoplasms of Three Different Histological Types. Auris Nasus Larynx, 34, 263-266. http://dx.doi.org/10.1016/j.anl.2006.07.001

[10] Keldahl, M.L., Zarif, A. and Gattuso, P. (2004) Bilateral Synchronous Pleomorphic Adenoma Diagnosed by FineNeedle Aspiration. Diagnostic Cytopathology, 30, 356-358. http://dx.doi.org/10.1002/dc.20030

[11] Kakimoto, N., Gamoh, S., Tamaki, J., et al. (2009) CT and MR Images of Pleomorphic Adenoma in Major and Minor Salivary Glands. European Journal of Radiology, 69, 464-472. http://dx.doi.org/10.1016/j.ejrad.2007.11.021

[12] Christe, A., Waldherr, C., Hallett, R., et al. (2011) MR Imaging of Parotid Tumors: Typical Lesion Characteristics in MR Imaging Improve Discrimination between Benign and Malignant Disease. American Journal of Neuroradiology, 32, 1202-1207. http://dx.doi.org/10.3174/ajnr.A2520

[13] Ghosh, S., Panarese, A., Bull, P.D., et al. (2003) Marginally Excised Parotid Pleomorphic Salivary Adenomas: Risk Factors for Recurrence and Management. A 12.5-Year Mean Follow-Up Study Histologically Marginal Excisions. Clinical Otolaryngology Allied Sciences, 28, 262-266. http://dx.doi.org/10.1046/j.1365-2273.2003.00704.X

[14] Brill, A.H. and Fitz-Hugh, G.S. (1975) Bilateral Mixed Tumors of the Parotid Gland. Archives of Otolaryngology, 101, 751-753. http://dx.doi.org/10.1001/archotol.1975.00780410043010 
Scientific Research Publishing (SCIRP) is one of the largest Open Access journal publishers. It is currently publishing more than 200 open access, online, peer-reviewed journals covering a wide range of academic disciplines. SCIRP serves the worldwide academic communities and contributes to the progress and application of science with its publication.

Other selected journals from SCIRP are listed as below. Submit your manuscript to us via either submit@scirp.org or Online Submission Portal.
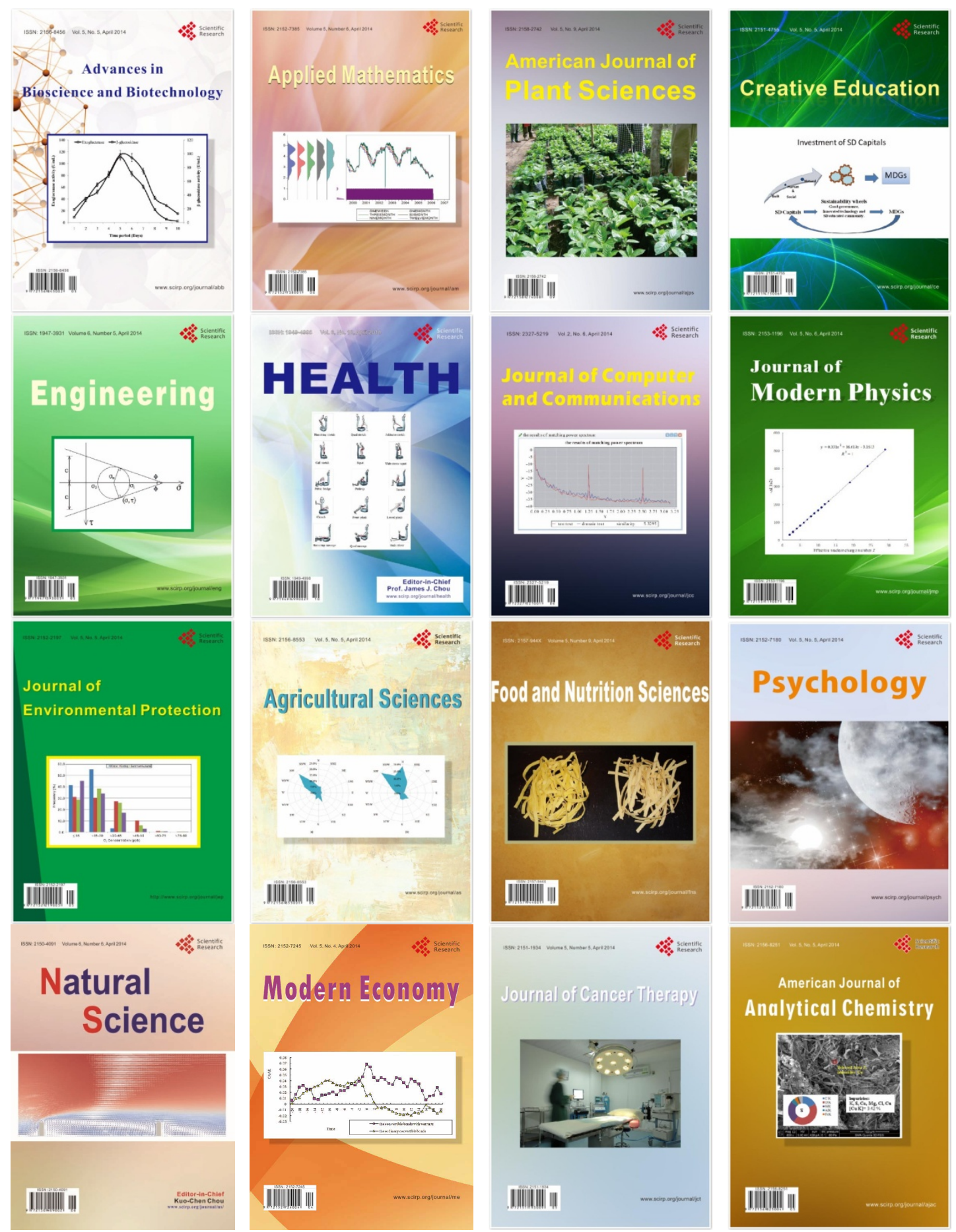